\title{
Modeling Non-homologous End Joining
}

\author{
Yongfeng Li \\ Division of Space Life Sciences, Universities Space Research Association
}

Non-homologous end joining (NHEJ) is the dominant DNA double strand break (DSB) repair pathway and involves several NHEJ proteins such as Ku, DNA-PKcs, XRCC4, Ligase IV and so on. Once DSBs are generated, $\mathrm{Ku}$ is first recruited to the DNA end, followed by other NHEJ proteins for DNA end processing and ligation. Because of the direct ligation of break ends without the need for a homologous template, NHEJ turns out to be an error-prone but efficient repair pathway. Some mechanisms have been proposed of how the efficiency of NHEJ repair is affected. The type of DNA damage is an important factor of NHEJ repair. For instance, the length of DNA fragment may determine the recruitment efficiency of NHEJ protein such as $\mathrm{Ku}$ [1], or the complexity of the DNA breaks [2] is accounted for the choice of NHEJ proteins and subpathway of NHEJ repair. On the other hand, the chromatin structure also plays a role of the accessibility of NHEJ protein to the DNA damage site. In this talk, some mathematical models of NHEJ, that consist of series of biochemical reactions complying with the laws of chemical reaction (e.g. mass action, etc.), will be introduced. By mathematical and numerical analysis and parameter estimation, the models are able to capture the qualitative biological features and show good agreement with experimental data. As conclusions, from the viewpoint of modeling, how the NHEJ proteins are recruited will be first discussed for connection between the classical sequential model [4] and recently proposed two-phase model [5]. Then how the NHEJ repair pathway is affected, by the length of DNA fragment [6], the complexity of DNA damage [7] and the chromatin structure [8], will be addressed.

\section{Reference}

[1] Wang H, Wang X, Zhang P, Wang Y (2008) The Ku-dependent non-homologous end-joining but not other repair pathway is inhibited by high linear energy transfer ionizing radiation. DNA repair 7: 725-733.

[2] Reynolds P, Anderson JA, Harper JV, Hill MA, Botchway SW, et al. (2012) The dynamics of Ku70/80 and DNAPKCs at DSBs induced by ionizing radiation is dependent on the complexity of damage. Nucleic acids research 40: 10821-10831. 
[3] Lorat Y, Schanz S, Schuler N, Wennemuth G, Rube C, et al. (2012) Beyond repair foci: DNA double-strand break repair in euchromatic and heterochromatic compartments analyzed by transmission electron microscopy.

PloS one 7: e38165.

[4] Mahaney BL, Meek K, Lees-Miller SP (2009) Repair of ionizing-induced DNA double-strand breaks by non-homologous end-joining. Biochem. J. 417, 639-650.

[5] Yano Y, Chen DJ (2008) Live cell imaging of XLF and XRCC4 reveals a oval view of protein assembly in the non-homologous end-joining pathway. Cell Cycle 7 (10), 1321-1325.

[6] Li Y, Qian H, Wang Y, Cucinotta FA (2012) A stochastic model of DNA fragments rejoining. PloS one 7: e44293

[7] Li Y, Reynolds P, O’Neill P, Cucinotta FA (2013) Modeling damage complexity-dependent nonhomologous end joining repair pathway, submitted

[8] Li Y and Cucinotta FA (2013) Modeling the Effects of Chromatin Structure on the non-homologous end joining, in preparation. 